\title{
Interferon-free treatment for hepatitis $C$ virus infection induces normalization of extrahepatic type I interferon signaling
}

\author{
Pil Soo Sung ${ }^{1,2, *}$, Eun Byul Lee ${ }^{1, *}$, Dong Jun Park ${ }^{1}$, Angelo Lozada ${ }^{3}$, Jeong Won Jang ${ }^{1,2}$, Si Hyun Bae ${ }^{1,2}$, \\ Jong Young Choi ${ }^{1,2}$, and Seung Kew Yoon ${ }^{1,2}$ \\ 'Liver Research Center, College of Medicine, The Catholic University of Korea, Seoul; ' 2 Department of Internal Medicine, College of \\ Medicine, The Catholic University of Korea, Seoul, Korea; ${ }^{3}$ Department of Internal Medicine, Makati Medical Center, Manila, Philippines
}

Background/Aims: Hepatitis C virus (HCV) replicates in the peripheral blood mononuclear cells (PBMCs), leading to the production of type I interferons (IFNs). It is well known that the gene expression profile of PBMC is similar to that of the liver. The present study explored the dynamic gene expression profile of PBMCs collected from HCV-infected patients undergoing direct-acting antiviral (DAA) therapy.

Methods: A prospective cohort comprising 27 patients under DAA therapy was formed. Expression level of IFN- $\beta$ and its downstream interferon-stimulated genes (ISGs) was measured in PBMCs before and after DAA treatment. Furthermore, immunoblotting was performed to identify the signaling molecules involved in the expression of ISGs.

Results: The pretreatment expression level of interferon-induced protein 44 (IFI44) and C-X-C motif chemokine ligand 10 (CXCL10) correlated with the pretreatment expression level of IFN- $\beta$. After DAA treatment, a significant decrease in the expression levels of IFN- $\beta$, IFI44, and CXCL10 was observed in the PBMCs. Furthermore, the pretreatment expression level of IFN- $\beta$ and ISGs correlated with the level of signal transducer and activator of transcription 1 (STAT1) phosphorylation, and DAA treatment abrogated STAT1 phosphorylation.

Conclusions: Pretreatment activation of IFN- $\beta$ response is rapidly normalized after DAA treatment. The present study suggests that the decreased type I IFN response by the clearance of HCV might contribute to DAA-induced alleviation of extrahepatic manifestation of chronic HCV infection. (Clin Mol Hepatol 2018;24:302-310)

Keywords: Hepatitis C virus; Antiviral agents; Interferon

\section{Study Highlights}

Hepatitis C virus (HCV) infection stimulates the induction of extrahepatic type I interferons (IFN), leading to the upregulation of interferon-stimulated genes. Pretreatment activation of extrahepatic type I IFN response is normalized after treatment of HCV infection with direct acting antivirals (DAAs). Our study suggests that the decreased type I IFN response by the clearance of HCV might contribute to DAA-induced alleviation of extrahepatic manifestation of chronic HCV infection.

\author{
Abbreviations: \\ ALT, alanine aminotransferase; CDNA, complementary DNA; CXCL10, C-X-C motif \\ chemokine ligand 10; DAA, direct-acting antiviral; GAPDH, glyceraldehyde \\ 3-phosphate dehydrogenase; HCV, hepatitis C virus; HRP, horseradish \\ peroxidase; IFI44, interferon-induced protein 44; IFN, interferon; ISG, interferon- \\ stimulated gene; JAK, Janus kinase; MAVS, mitochondrial antiviral signaling \\ protein; Mx1, myxovirus resistance-1; NS5A, non-structural 5A; PBMC, \\ peripheral blood mononuclear cell; PCR, polymerase chain reaction; PY-STAT1, \\ tyrosine-phosphorylated STAT1; RAS, resistance-associated substitution; RIPA, \\ radioimmunoprecipitation assay; SOF, sofosbuvir; STAT1, signal transducer and \\ activator of transcription 1; SVR, sustained virologic response; TRIF, Toll/IL-1 \\ receptor domain-containing adaptor inducing IFN- $\beta$

Corresponding author: Seung Kew Yoon

Department of Internal Medicine, College of Medicine, The Catholic University of Korea, 222 Banpo-daero, Seocho-gu, Seoul 06591, Korea Tel: +82-2-2258-1425, Fax: +82-2-3481-4025

E-mail:yoonsk@catholic.ac.kr

http://orcid.org/0000-0002-4476-4868 


\section{INTRODUCTION}

Hepatitis C virus (HCV) is a positive-stranded RNA virus, and more than 184 million people are infected with HCV worldwide.' After HCV infection, most of the patients fail to clear the virus from the blood and develop chronic persistent infection. ${ }^{2}$ Furthermore, HCV infection often leads to the development of hepatic complications, such as cirrhosis and hepatocellular carcinoma. Up to $70 \%$ of the patients with chronic HCV infection experience extrahepatic manifestations. $^{3}$

Many types of interferons (IFNs) have been identified. ${ }^{4,5}$ Upon binding of IFNs to their respective receptors, cellular actions are mediated through specific interferon-stimulated genes (ISGs) with antiviral and immunoregulatory effects. ${ }^{5}$ Currently, IFNs are classified into three major classes: type I, type II, and type III. Each class of IFNs signals to the host cell by binding to different receptor complexes. Type I IFN comprises 13 IFN- $\alpha$, in addition to IFN- $\beta$, IFN- $\omega$, IFN- $\varepsilon$, and IFN- $\kappa{ }^{5}$ Type I IFNs are critical mediators of inflammation and immunosuppression during chronic viral infection. ${ }^{6}$ The control of these infections requires type I IFN, although prolonged exposure results in the dysfunction of immune cells. ${ }^{6}$

After the establishment of HCV infection, type I and III IFNs are endogenously produced by the HCV-infected cells. ${ }^{7}$ Consequently, type I and III IFNs after binding to their respective receptors initiate a signaling cascade through the Janus kinase (JAK)-signal transducer and activator of transcription (STAT) pathway. ${ }^{7,8}$ The cellular actions are mediated by the induction of ISGs. Our recent study demonstrated that endogenous type I and III IFNs are produced in response to HCV infection, thus rendering the cells nonresponsive to exogenous IFN- $\alpha^{9,10}$ Furthermore, treating the HCV-infected primary human hepatocytes with telaprevir and sofosbuvir (SOF) reduced the intracellular HCV RNA titer resulting in the attenuated induction of ISGs and restoration of IFN- $\alpha$ responsiveness. ${ }^{10}$

Previous studies have reported that HCV can replicate in the peripheral blood mononuclear cells (PBMCs). ${ }^{11-13}$ A recent study reported that G28A variant HCV replicates in the PBMCs independent of microRNA (mir)-122. ${ }^{11}$ It has also been shown that this variant is associated with extrahepatic manifestation of chronic HCV infection." Furthermore, another report showed that the pattern of ISG expression is similar in the liver and PBMCs of HCV-infected patients. ${ }^{14}$ These reports suggest that HCV can replicate in the PBMCs, and induce a pattern of ISG expression similar to that in the liver.

Recently, various types of direct-acting antivirals (DAAs) against HCV have been developed. A high rate of sustained virologic response (SVR) is achieved by DAAs without the addition of IFNs. ${ }^{15}$
As a result, the standard of care for chronic HCV infection has shifted from IFN $\alpha$-based therapies to DAAs. ${ }^{16}$ The present study explored the dynamic gene expression profile of PBMCs collected from HCV-infected patients treated with DAA therapy. The expression level of IFN- $\beta$ and ISGs was analyzed before and after DAA treatment, and correlated the expression level with the level of STAT1 phosphorylation and clinical parameters.

\section{PATIENTS AND METHODS}

\section{Patients}

From March 1 to September 30 2016, 51 patients with HCV genotype $1 \mathrm{~b}$ infection receiving dual oral therapy with daclatasvir (Daklinza ${ }^{\circledR}$, Bristol Myers Squibb, New York, NY, USA) and asunaprevir (Sunvepra $^{\circledR}$, Bristol Myers Squibb, New York, NY, USA) for 24 weeks (wks) at the Division of Hepatology in the Seoul St. Mary's Hospital were examined. Among them, 21 patients agreed to donate blood samples and were enrolled in the present study. Five patients infected with HCV genotype 2 were also enrolled. They were treated with SOF (Sovaldi ${ }^{\circledR}$, Gilead, Foster City, CA, USA) and ribavirin. One patient infected with HCV genotype 1a was enrolled and was treated with ledipasvir plus SOF (Harvoni ${ }^{\circledR}$, Gilead, Foster City, CA, USA).

A prospective cohort with these 27 patients was formed, blood samples were obtained, and PBMCs were isolated at the start and end of the treatment. Detailed history taking and physical examination were performed to confirm extrahepatic manifestation of $\mathrm{HCV}$ in the enrolled patients. Liver stiffness was measured by transient elastography using the FibroScan ${ }^{\circledR}$ (Echosens, Paris, France), and the result was expressed in $\mathrm{kPa}$. For patients infected with HCV genotype 1, non-structural 5A protein (NS5A) resistance-associated substitution (RAS) testing was performed. ${ }^{17}$ Population sequencing (Sanger method) of the full-length HCV NS5A coding region was performed by Seoul Clinical Laboratories (Yongin, Korea). Further, NS5A RASs were defined as the following substitutions at the following positions: L31//F/M/V and Y93C/F/H/N/S.

SVR12 was defined as undetectable HCV RNA at 12 wk after the completion of treatment. Virologic failure consisted of viral breakthrough (detectable on treatment with HCV RNA following an undetectable level), relapse (undetectable HCV RNA at the end of treatment, and then quantifiable $12 \mathrm{wk}$ after the end of treatment), and undefined failure (detectable HCV RNA level during all the visits). The HCV RNA copy number in the sera of the enrolled patients was determined. 
The present study was conducted according to the Declaration of Helsinki principles and was approved by the institutional review boards (Seoul St. Mary's Hospital, KC150ISI0787). The written informed consent was received from the participants prior to their inclusion in the study.

\section{RNA extraction, complementary DNA synthesis, and real-time quantitative polymerase chain reaction analysis}

The PBMCs were isolated from patients with chronic HCV infection by Ficoll-Hypaque density gradient method. ${ }^{18}$ Total RNA isolation, complementary DNA (cDNA) synthesis, and real-time quantitative polymerase chain reaction (PCR) analysis were performed as previously described. ${ }^{19}$ Real-time quantitative PCR with $5^{\prime}$-nuclease probes is a relatively robust and precise method for specific DNA sequence detection and quantification with minimal background signal. ${ }^{20}$ TaqMan Gene Expression Assays (Applied Biosystems, Foster City, CA, USA) were used to determine the level of mRNA of the target genes. The assay ID of each gene is as follows: IFI44 (Hs00197427_m1), CXCL10 (Hs00171042_m1), IFN- $\beta$ (Hs01077958_s1), and glyceraldehyde 3-phosphate dehydrogenase (GAPDH) (Hs02786624_g1). Real-time quantitative PCR was performed with the following temperature settings: (1) $95^{\circ} \mathrm{C}$ for $10 \mathrm{~min}$, and (2) 40 cycles at $95^{\circ} \mathrm{C}$ for $15 \mathrm{~s}$, and $60^{\circ} \mathrm{C}$ for $30 \mathrm{~s}$. The results were standardized to the mRNA level of GAPDH, and the data were presented as the mean \pm standard deviation (SD). To quantify HCV RNA, previously reported and validated primers and probes were used. ${ }^{19}$

\section{Immunoblotting}

The detailed immunoblotting protocol has been described previously. ${ }^{21}$ Pellet of PBMCs was lysed with radioimmunoprecipitation assay (RIPA) buffer, and $7 \mu \mathrm{g}$ of the cell lysate was loaded onto sodium dodecyl sulfate-polyacrylamide gels. The blotted membrane was blocked in 5\% non-fat dry milk diluted in tris-buffered saline for $1.5 \mathrm{~h}$ at room temperature. Primary antibodies were diluted in the blocking buffer, and the membrane was incubated with the diluted primary antibodies for $19 \mathrm{~h}$ at $4^{\circ} \mathrm{C}$. After washing, the membrane was incubated with horseradish peroxidase (HRP)-conjugated secondary antibodies for $30 \mathrm{~min}$ at room temperature. After washing, the ImageQuant LAS 4000 (GE Healthcare, Hatfield, UK) was used to obtain the images. The antibodies used are as follows: STAT1 (1:1,000, rabbit, BD transduction laboratories, San Jose, CA, USA),
PY-STAT1 (1:1,000, mouse, BD transduction laboratories), GAPDH (1:1,000, rabbit, Santa Cruz Biotechnology, Santa Cruz, CA, USA), HRP-conjugated rabbit immunoglobulin G (IgG) (1:5000, Santa Cruz Biotechnology, Santa Cruz, CA, USA), and HRP-conjugated mouse IgG (1:5,000, Santa Cruz Biotechnology, Santa Cruz, CA, USA).

\section{Statistical analyses}

The discrete variables were compared by the $\chi^{2}$ test, and independent $t$-test was used for continuous variables. Both Pearson and Spearman correlation analyses were used to evaluate the correlation between continuous variables. Statistical significance was defined as a $P$ value $<0.05$. SPSS version 20 software (IBM Corp., Armonk, NY, USA) was used for all the analyses.

\section{RESULTS}

\section{Study population}

Patient demographics were summarized in Table 1. The median

Table 1. Baseline characteristics of the patients

\begin{tabular}{lc}
\hline Variable & Value $(\mathbf{n}=\mathbf{2 7})$ \\
\hline Age, years & $63(34-75)$ \\
Male sex, $n(\%)$ & $9(33)$ \\
\hline HCV genotype & $1(3.7)$ \\
$1 \mathrm{a}$ & $21(77.8)$ \\
$1 \mathrm{~b}$ & $5(18.5)$ \\
2 & $475,000(165-21,200,000)$ \\
HCV RNA, IU/mL & $0(0)$ \\
HCV NS5A RAS & $35(17-166)$ \\
AST, U/L & $32(14-300)$ \\
ALT, U/L & $23(85)$ \\
Chronic hepatitis, $n$ (\%) & $4(15)$ \\
Compensated cirrhosis, $n(\%)$ & \\
Prior HCV therapy, $n(\%)$ & $20(74)$ \\
Treatment-naive & $2(7)$ \\
Non-responders & $2(7)$ \\
Relapsers & $3(1)$ \\
IFN/RBV intolerant & $6.7(3.0-39.7)$ \\
\hline Liver stiffness, KPa &
\end{tabular}

Values are presented as $\mathrm{n}(\%)$ or $\mathrm{n}$ (median range) unless otherwise indicated.

HCV, hepatitis C virus; NS5A, non-structural 5A protein; RAS, resistance-associated substitution; AST, aspartate transaminase; ALT, alanine aminotransferase; IFN, interferon; RBV, ribavirin. 
age of the patients was 63 years. Non-structural 5A protein RASs were not detected in any of the enrolled patients. Among the patients, 20 patients were treatment-naïve. Two patients were nonresponders and two patients relapsed after treatment with the pegylated IFN plus ribavirin. Three patients were intolerant to the pegylated IFN plus ribavirin treatment and did not complete the schedule. Four patients were diagnosed with liver cirrhosis and 23 patients were diagnosed with chronic hepatitis. The median value of liver stiffness measured by transient elastography was $6.7 \mathrm{kPa}$. At least one clinical extrahepatic manifestation was observed in 17 of 27 patients $(63 \%)$. The most common extrahepatic manifestation was fatigue (33\%). Type II diabetes (15\%), arthralgia (15\%), pruritus (11\%), depressive mood (11\%), chronic kidney diseases $(7 \%)$, and sicca syndrome (4\%) were also observed in the study group. The SVR12 rate was $90.5 \%$ (19/21) in patients with HCV genotype $1 \mathrm{~b}$ infection, 100\% (1/1) in patients with HCV genotype 1a infection, and 100\% (5/5) in patients with HCV genotype 2 infection.

\section{Treatment-induced downregulation of endogenous IFN- $\beta$ signaling in the PBMCs of HCV-infected patients}

Initially, the mRNA level of IFN- $\beta$, IFI44, and CXCL10 in the PBMCS of the cohort before DAA treatment was measured. Further, IFI44 and CXCL10 were chosen, because they were proved as representative ISGs induced by IFN- $\beta$ in our previous studies. ${ }^{2,9,10}$ The pretreatment expression level of IFI44 and CXCL10 correlated with the pretreatment expression level of IFN- $\beta$ (Fig. 1A). However, the expression level of these ISGs was not associated with the expression of IFN- $\lambda 1$ (data not shown).

The present study also verified if the mRNA level of IFN- $\beta$, IFI44, and CXCL10 in PBMCs before treatment correlated with the baseline HCV RNA copies, alanine aminotransferase (ALT) level, or liver stiffness. The results revealed that the pretreatment expression level of IFN- $\beta$, IFI44, and CXCL10 did not correlate with the baseline HCV RNA copies (Fig. 1B), ALT levels (Fig. 1C), or liver stiffness (Fig. 1D).

Subsequently, the mRNA level of IFN- $\beta$, IFI44, and CXCL10 in the PBMCs before DAA treatment was compared with those after DAA treatment. The expression level of IFN- $\beta$ was significantly downregulated in the PBMCs after DAA treatment (Fig. 2A). Similarly, the expression level of IFI44 and CXCL10 was also downregulated in the PBMCs after DAA treatment (Fig. 2B). Viral kinetics of the enrolled patients was presented in Fig. 2 C. There were two patients with viral breakthrough (Fig. 2C), and they did not show a decrease in the expression level of these genes (Fig. 2D).

\section{Level of STAT1 phosphorylation decreased after DAA treatment in the PBMCs of $\mathrm{HCV}$-infected patients}

To confirm the decreased activation of JAK-STAT signaling by DAA treatment, immunoblotting of STAT1 and tyrosine-phosphorylated STAT1 (PY-STAT1) proteins was performed using the PBMCs of patients. As expected, the level of STAT1 phosphorylation before DAA treatment correlated with the mRNA levels of CXCL10 $(P=0.03$, $\mathrm{R}=0.86$ ) (Fig. 3A, B). However, the level of STAT1 phosphorylation was not significantly associated with IFI44. After DAA treatment, the level of STAT1 phosphorylation decreased (Fig. 3C). These results were presented in Fig. 2, which showed the decreased production of IFN- $\beta$ after DAA treatment. Overall, the endogenous IFN- $\beta$ signaling, represented by STAT1 phosphorylation and ISGS induction, was downregulated by DAA treatment in the PBMCs collected from HCV-infected patients.

\section{DISCUSSION}

The present study demonstrated that the pretreatment activation of IFN- $\beta$ response, reflected by the upregulation of ISGs transcripts and level of STAT1 phosphorylation, is rapidly normalized after DAA treatment.

In HCV-infected cells, double-stranded viral RNA is sensed by retinoic acid-inducible gene I, melanoma differentiation-associated protein 5 , and Toll-like receptor 3 , thus activating downstream signaling and inducing type I IFNs. ${ }^{2,22}$ Intracellular signals from the receptors are transmitted to mitochondrial antiviral signaling protein (MAVS) and Toll/IL-1 receptor domain-containing adaptor inducing IFN- $\beta$ (TRIF), followed by the induction of IFNs. ${ }^{22,23}$ Further, HCV uses mechanisms to interfere with the induction of IFNs. Importantly, HCV NS3/4A protease is known to cleave MAVS and TRIF, consequently blocking the transmission of the intracellular signaling toward type I IFN production. ${ }^{2}$ However, HCV cannot completely block the signaling, and it has been demonstrated that HCV infection is associated with the activation of the type I IFN system by the induction of ISGs. ${ }^{24,25}$ Continuous upregulation of ISGs in the HCVinfected liver has been reported in chimpanzee models ${ }^{24,26}$ and HCV-infected humans. ${ }^{27,28}$ Interestingly, HCV RNA and ISG mRNA are detected simultaneously in the hepatocytes of patients with chronic HCV infection. ${ }^{29}$ These findings suggest that HCV infection stimulates the induction of endogenous IFNs, which result in the up- 


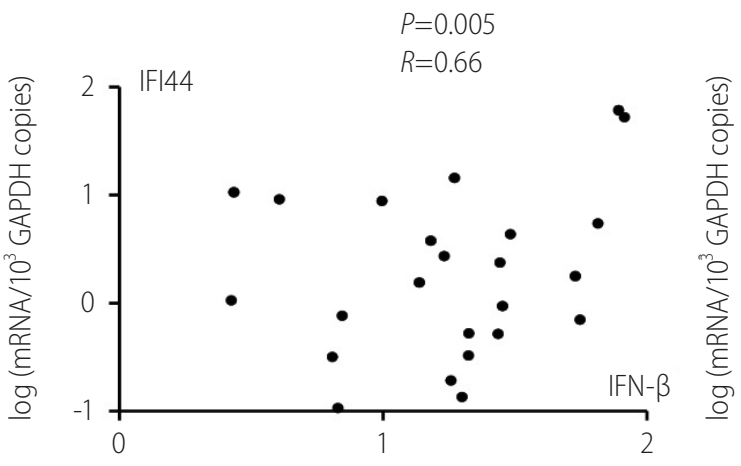

A $\log$ (mRNA copies/10 GAPDH copies)

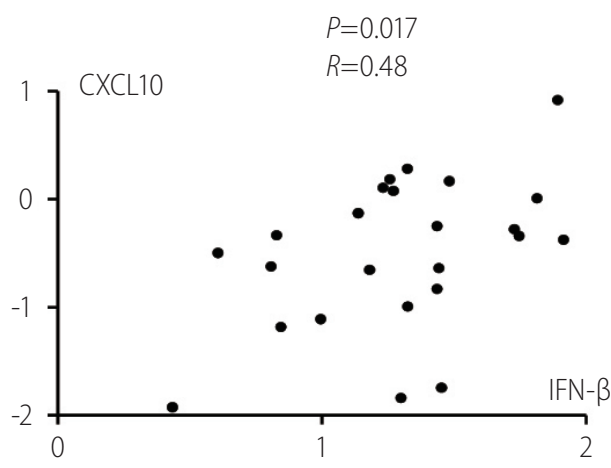

$\log$ (mRNA copies $/ 10^{3}$ GAPDH copies)

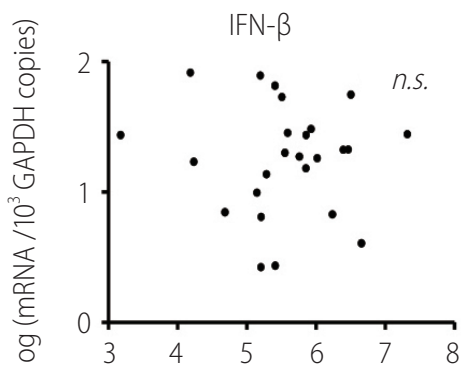

B $\quad \log (\mathrm{HCV} R \mathrm{NA} / \mathrm{mL})$
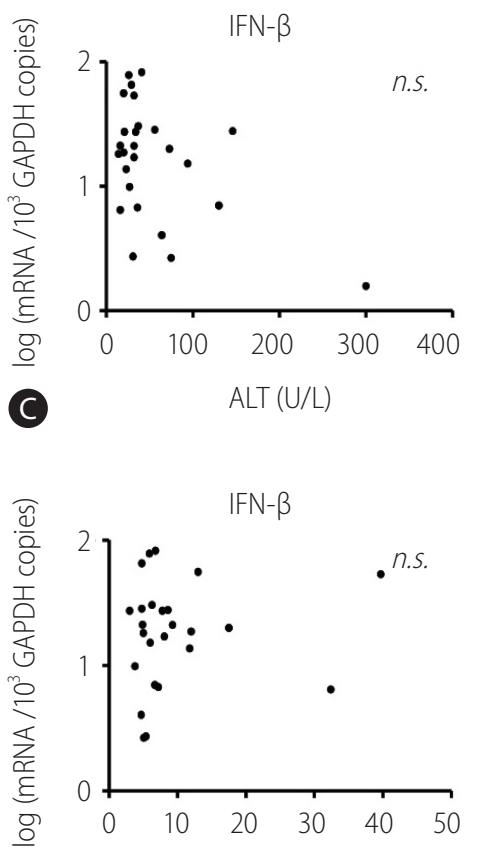

(D)

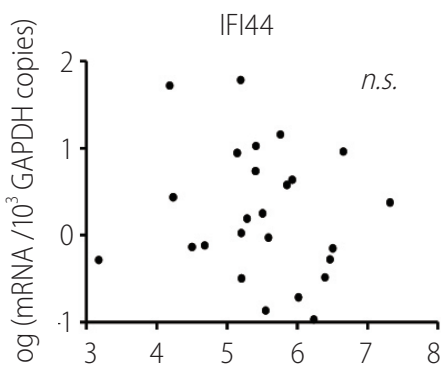

$\log (\mathrm{HCV} R \mathrm{RA} / \mathrm{mL})$

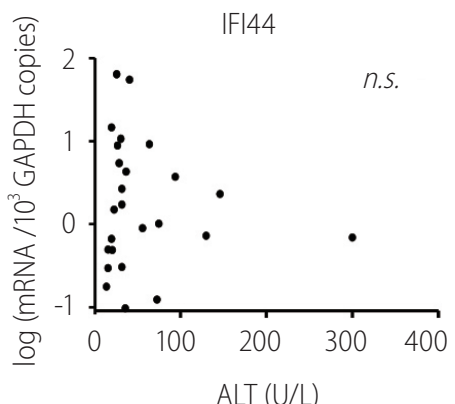

IFI44

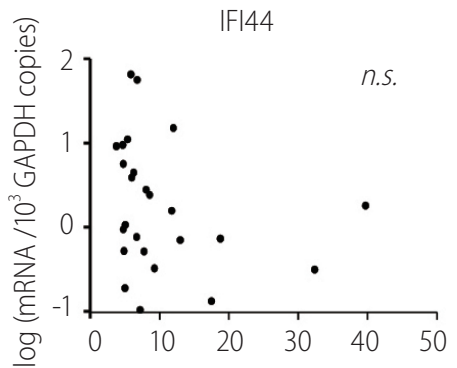

Liver Stiffness (kPa)

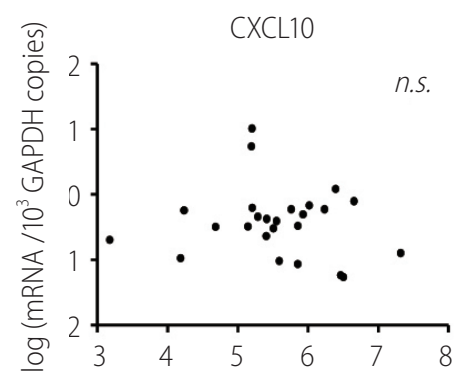

$\log (\mathrm{HCV} R \mathrm{RN} / \mathrm{mL})$
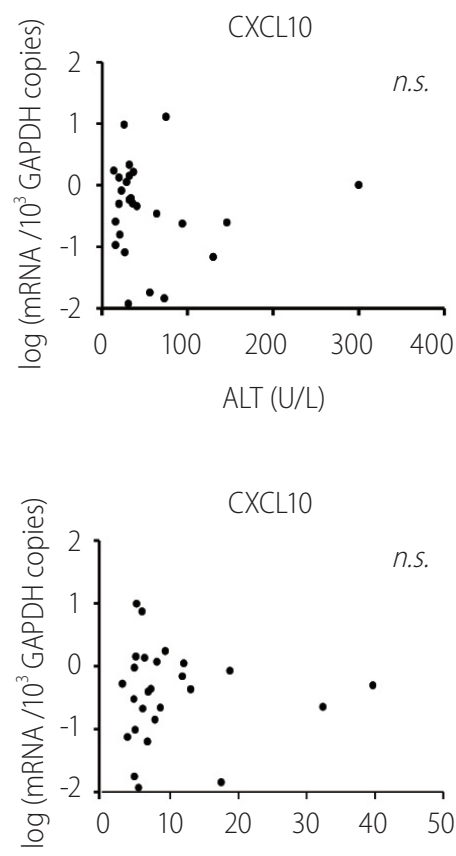

Liver Stiffness ( $\mathrm{kPa}$ )

Figure 1. Pretreatment IFN- $\beta$ expression correlates with the expression of IFI44 and CXCL10 in the peripheral blood mononuclear cells (PBMCs) isolated from HCV-infected patients. (A-D) PBMCs from HCV-infected patients were isolated before direct-acting antiviral treatment. TaqMan real-time quantitative polymerase chain reaction was performed to detect mRNA levels of IFN- $\beta$, IFI44, CXCL10, and GAPDH. Pearson's correlation analysis was performed. GAPDH, glyceraldehyde 3-phosphate dehydrogenase; IFI44, interferon-induced protein 44; IFN, interferon; CXCL10, C-X-C motif chemokine ligand 10; n.S., not significant; HCV, hepatitis C virus; ALT, alanine aminotransferase. 

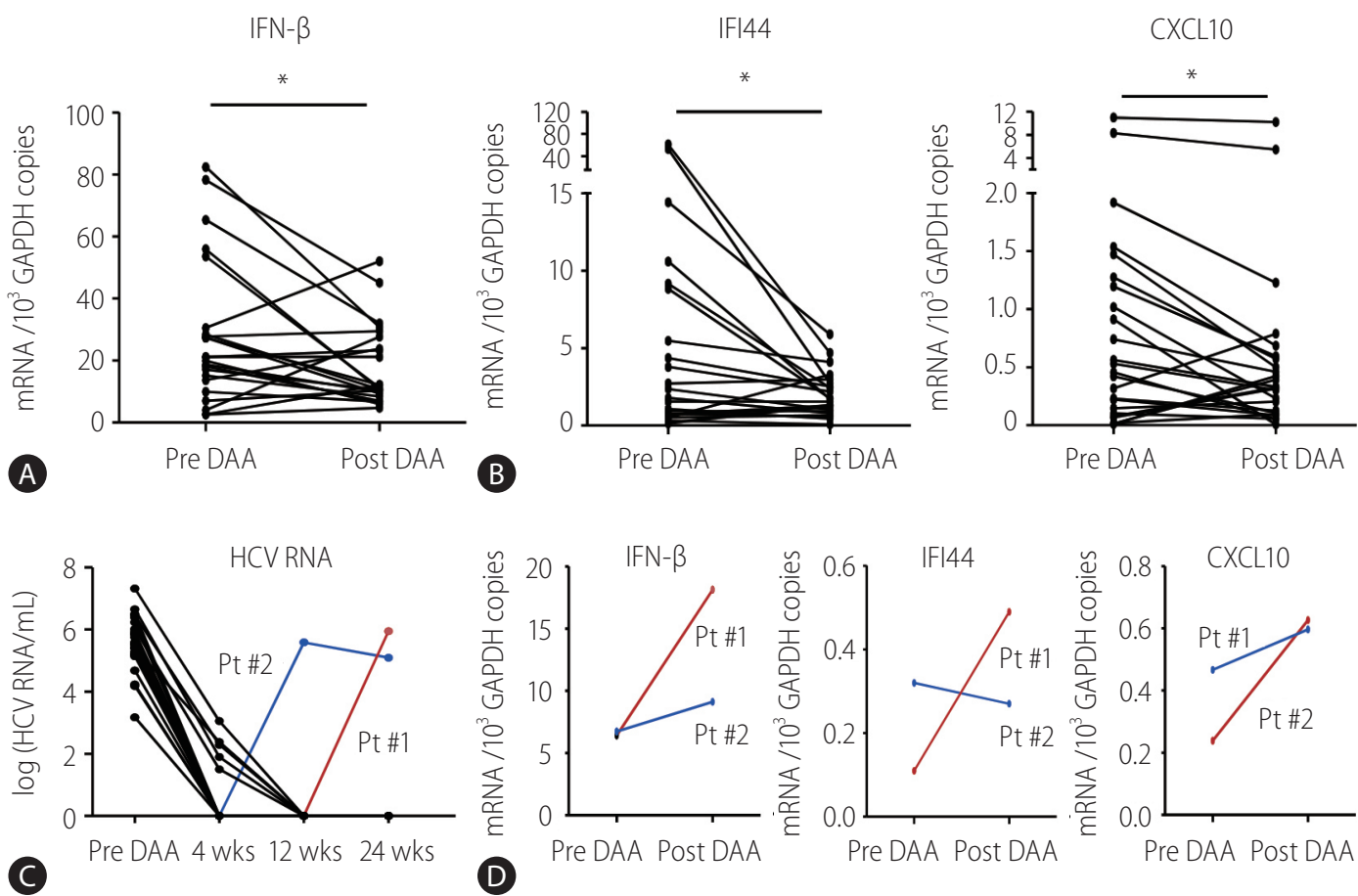

Figure 2. Direct-acting antiviral (DAA)-induced downregulation of endogenous IFN- $\beta$ response in peripheral blood mononuclear cells (PBMCs) isolated from HCV-infected patients. (A, B) PBMCs from HCV-infected patients were isolated before DAA (pre DAA) and at the end of DAA (post DAA) treatment. TaqMan real-time quantitative polymerase chain reaction (PCR) was performed to detect mRNA levels of IFN- $\beta$, IFI44, CXCL10, and GAPDH. (C, D) Serum and PBMC from two HCV-infected patients were isolated before and th the end of DAA treatment. TaqMan real-time quantitative PCR was performed to detect viral RNA level of HCV in the sera and mRNA levels of IFN- $\beta$, IFI44, CXCL10, and GAPDH in the PBMCs. GAPDH, glyceraldehyde 3-phosphate dehydrogenase; IFN, interferon; IFI44, interferon-induced protein 44; CXCL10, C-X-C motif chemokine ligand 10; HCV, hepatitis C virus; wks, weeks; Pt, patient. ${ }^{*} P<0.05$.

regulation of ISGs in the infected liver. ${ }^{29}$ Because a previous study reported that the expression pattern of ISGs is similar in the liver and PBMCs of HCV-infected patients, ${ }^{14}$ the present study used the PBMCs of patients to monitor changes in the expression of IFN- $\beta$ and the induction of downstream ISGs.

Our previous study demonstrated that the expression of CXCL10 is more rapidly induced and better correlates with STAT1 phosphorylation by type I and II IFNs than that of other antiviral ISGS, such as IFI44, IFI27, or myxovirus resistance-1 (Mx1). 10,21 The results of the present study showed that the expression of CXCL10 correlated with the level of STAT1 phosphorylation. However, the expression of IFI44 did not correlate with the level of STAT1 phosphorylation (Fig. 3B). The lack of correlation between the expression of IFI44 and the level of STAT1 phosphorylation might be attributed to the limited number of patients enrolled.

Hepatitis C virus infection is associated with the development of various extrahepatic manifestations. ${ }^{3}$ They might partly stem from the HCV infection to various types of PBMCs, such as monocytes, dendritic cells, and lymphocytes ${ }^{11}$ leading to the activation of type I
IFN response in these cells. ${ }^{30}$ Eradication of virus with DAA is associated with a reduction not only in liver-related events of HCV infection, but also in HCV-related extrahepatic manifestations. ${ }^{31} \mathrm{~A}$ recent report showed that IFNL4 genotype (previously known as IL28B genotype) influences the baseline ISG expression level in the PBMCS of HCV-infected patients suggesting a functional link between the IFNL4 genetic variation and immune response in cells of extrahepatic origin. ${ }^{32}$ Given that the HCV-infected patients with cryoglobulinemic vasculitis ${ }^{33}$ and metabolic extrahepatic manifestations $^{34}$ have higher frequency of IL-28B T/T genotype, which is proven to cause high intrahepatic baseline ISG levels, ${ }^{2,7,9}$ a decreased expression of extrahepatic ISGs by DAAs observed in the present study might partly explain the alleviation of extrahepatic manifestations after DAA treatment.

The present study had some limitations: (1) the expression of ISGS was evaluated in unstimulated PBMC, which might not reflect the true activity of IFN in the liver, (2) the analyses were performed using unsorted PBMCs, which might lead to confusion during data analyses, and (3) the relatively small number of patients enrolled 


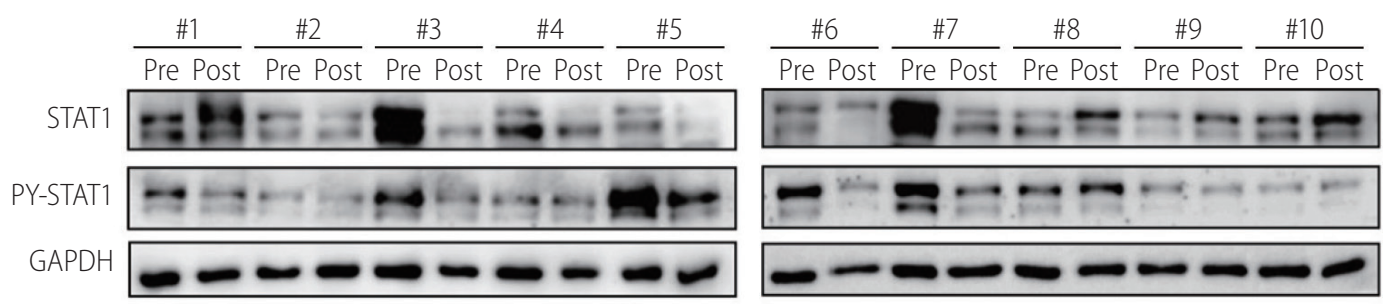

A
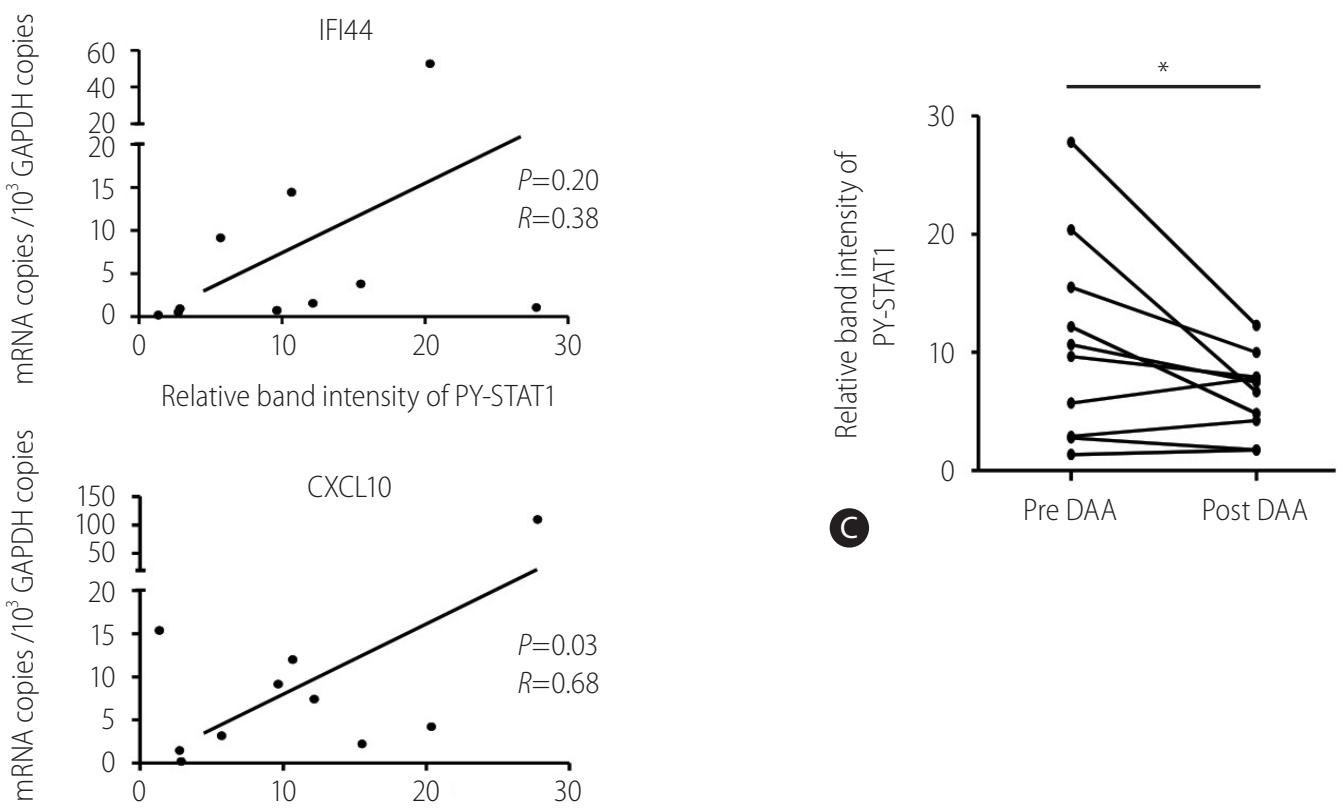

B Relative band intensity of PY-STAT1

Figure 3. STAT1 phosphorylation decreased in the peripheral blood mononuclear cells (PBMCs) of HCV-infected patients after direct-acting antiviral (DAA) treatment. (A, C) PBMCs from HCV-infected patients were isolated before DAA (pre DAA) and at the end of DAA (post DAA) treatment. Immunoblotting was performed to detect the protein levels of STAT1, PY-STAT1, and GAPDH. (B) PBMCs from HCV-infected patients were isolated before DAA treatment. TaqMan real-time quantitative polymerase chain reaction was performed to detect mRNA levels of IFI44, CXCL10, and GAPDH. Pearson's correlation analysis was performed to identify the associations between expression levels of IFI44/CXCL10 and relative band intensity of PY-STAT1. STAT1, signal transducer and activator of transcription 1; HCV, hepatitis C virus; PY-STAT1, tyrosine-phosphorylated STAT1; GAPDH, glyceraldehyde 3-phosphate dehydrogenase; IFI44, interferon-induced protein 44; CXCL10, C-X-C motif chemokine ligand 10. ${ }^{*} P<0.05$.

limited the analyses.

The present study demonstrated that the pretreatment activation of IFN- $\beta$ response is normalized after DAA treatment. The results suggest that the decreased expression of ISGs might be one of the mechanisms of DAA-induced alleviation of extrahepatic manifestations during $\mathrm{HCV}$ infection.

\section{Author contributions}

P.S.S., E.B.L, and S.K.Y. designed and carried out the study; P.S.S., E.B.L, D.J.P., A.L., J.W.J., S.H.B., J.Y.C., and S.K.Y. analyzed the data; P.S.S. and S.K.Y. wrote the paper.

\section{Acknowledgments}

The present study was partly supported by the Global Hightech Biomedicine Technology Development Program of the National Research Foundation (NRF) and Korea Health Industry Development Institute (KHIDI) funded by the Korean government (MSIP \& MOHW) (No. 2015M3D6A1065146). This research was also supported by the Basic Science Research Program through the National Research Foundation of Korea (NRF) funded by the Ministry of Education (NRF-2017R1D1A1B03033718). 


\section{Conflicts of Interest}

The authors declare no financial conflict of interest.

\section{REFERENCES}

1. Thrift AP, El-Serag HB, Kanwal F. Global epidemiology and burden of HCV infection and HCV-related disease. Nat Rev Gastroenterol Hepatol 2017;14:122-132.

2. Shin EC, Sung PS, Park SH. Immune responses and immunopathology in acute and chronic viral hepatitis. Nat Rev Immunol 2016;16:509523.

3. Ramos-Casals M, Zignego AL, Ferri C, Brito-Zerón P, Retamozo S, Casato $\mathrm{M}$, et al. Evidence-based recommendations on the management of extrahepatic manifestations of chronic hepatitis C virus infection. J Hepatol 2017;66:1282-1299.

4. Stark GR, Darnell JE Jr. The JAK-STAT pathway at twenty. Immunity 2012;36:503-514.

5. Borden EC, Sen GC, Uze G, Silverman RH, Ransohoff RM, Foster GR, et al. Interferons at age 50: past, current and future impact on biomedicine. Nat Rev Drug Discov 2007;6:975-990.

6. Snell LM, McGaha TL, Brooks DG. Type I interferon in chronic virus infection and cancer. Trends Immunol 2017;38:542-557.

7. Sung PS, Shin EC, Yoon SK. Interferon response in hepatitis $C$ virus (HCV) infection: lessons from cell culture systems of HCV infection. Int J Mol Sci 2015;16:23683-23694.

8. Snell LM, Brooks DG. New insights into type I interferon and the immunopathogenesis of persistent viral infections. Curr Opin Immunol 2015;34:91-98.

9. Sung PS, Cheon $\mathrm{H}$, Cho CH, Hong SH, Park DY, Seo HI, et al. Roles of unphosphorylated ISGF3 in HCV infection and interferon responsiveness. Proc Natl Acad Sci U S A 2015;112:10443-10448.

10. Sung PS, Hong SH, Chung JH, Kim S, Park SH, Kim HM, et al. IFN- $\lambda 4$ potently blocks IFN- $\alpha$ signalling by ISG15 and USP18 in hepatitis C virus infection. Sci Rep 2017;7:3821.

11. Ono C, Fukuhara T, Motooka D, Nakamura S, Okuzaki D, Yamamoto S, et al. Characterization of miR-122-independent propagation of HCV. PLoS Pathog 2017;13:e1006374.

12. Petersen $T$, Lee YJ, Osinusi A, Amorosa VK, Wang C, Kang M, et al. Interferon stimulated gene expression in HIV/HCV coinfected patients treated with nitazoxanide/peginterferon-Alfa-2a and ribavirin. AIDS Res Hum Retroviruses 2016;32:660-667.

13. Radkowski M, Opoka-Kegler J, Cortes KC, Bukowska-Ośko I, Perlejewski K, Pawełczyk A, et al. Evidence for immune activation in patients with residual hepatitis $C$ virus RNA long after successful treatment with IFN and ribavirin. J Gen Virol 2014;95(Pt 9):2004-2009.

14. Meissner EG, Wu D, Osinusi A, Bon D, Virtaneva K, Sturdevant D, et al. Endogenous intrahepatic IFNs and association with IFN-free HCV treatment outcome. J Clin Invest 2014;124:3352-3363.

15. Korean Association for the Study of the Liver. KASL clinical practice guidelines: management of hepatitis C. Clin Mol Hepatol 2016;22:76139.

16. Rosen HR. "Hep C, where art thou": What are the remaining (fundable) questions in hepatitis C virus research? Hepatology 2017;65:341-349.

17. Sarrazin C, Dvory-Sobol H, Svarovskaia ES, Doehle BP, Pang PS, Chuang $S M$, et al. Prevalence of resistance-associated substitutions in HCV NS5A, NS5B, or NS3 and outcomes of treatment with ledipasvir and sofosbuvir. Gastroenterology 2016;151:501-512.e1.

18. Seong YJ, Sung PS, Jang YS, Choi YJ, Park BC, Park SH, et al. Activation of human natural killer cells by the soluble form of cellular prion protein. Biochem Biophys Res Commun 2015;464:512-518.

19. Sung PS, Murayama A, Kang W, Kim MS, Yoon SK, Fukasawa M, et al. Hepatitis $C$ virus entry is impaired by claudin-1 downregulation in diacylglycerol acyltransferase-1-deficient cells. J Virol 2014;88:92339244.

20. Holland PM, Abramson RD, Watson R, Gelfand DH. Detection of specific polymerase chain reaction product by utilizing the $5^{\prime}----3^{\prime}$ exonuclease activity of Thermus aquaticus DNA polymerase. Proc Natl Acad Sci U S A 1991;88:7276-7280.

21. Sung PS, Hong SH, Lee J, Park SH, Yoon SK, Chung WJ, et al. CXCL10 is produced in hepatitis A virus-infected cells in an IRF3-dependent but IFN-independent manner. Sci Rep 2017;7:6387.

22. Park SH, Rehermann B. Immune responses to HCV and other hepatitis viruses. Immunity 2014;40:13-24.

23. Horner SM, Gale M Jr. Regulation of hepatic innate immunity by hepatitis C virus. Nat Med 2013;19:879-888.

24. Su Al, Pezacki JP, Wodicka L, Brideau AD, Supekova L, Thimme R, et al. Genomic analysis of the host response to hepatitis $C$ virus infection. Proc Natl Acad Sci U S A 2002;99:15669-15674.

25. Lanford RE, Feng Z, Chavez D, Guerra B, Brasky KM, Zhou Y, et al. Acute hepatitis $A$ virus infection is associated with a limited type I interferon response and persistence of intrahepatic viral RNA. Proc Natl Acad Sci U S A 2011;108:11223-11228.

26. Wieland SF, Chisari FV. Stealth and cunning: hepatitis B and hepatitis C viruses. J Virol 2005;79:9369-9380.

27. Dill MT, Duong FH, Vogt JE, Bibert S, Bochud PY, Terracciano L, et al. Interferon-induced gene expression is a stronger predictor of treatment response than IL28B genotype in patients with hepatitis C. Gastroenterology 2011;140:1021-1031.

28. Sarasin-Filipowicz M, Oakeley EJ, Duong FH, Christen V, Terracciano $L$, Filipowicz W, et al. Interferon signaling and treatment outcome in chronic hepatitis C. Proc Natl Acad Sci U S A 2008;105:7034-7039.

29. Wieland S, Makowska Z, Campana B, Calabrese D, Dill MT, Chung $J$, et al. Simultaneous detection of hepatitis $C$ virus and interferon stimulated gene expression in infected human liver. Hepatology 
2014;59:2121-2130.

30. Griesbeck M, Valantin MA, Lacombe K, Samri-Hassimi A, Bottero J, Blanc $C$, et al. Hepatitis C virus drives increased type I interferon-associated impairments associated with fibrosis severity in antiretroviral treatment-treated HIV-1-hepatitis C virus-coinfected individuals. AIDS 2017;31:1223-1234.

31. van der Meer AJ, Berenguer M. Reversion of disease manifestations after HCV eradication. J Hepatol 2016;65(1 Suppl):S95-S108.

32. Rosenberg BR, Freije CA, Imanaka N, Chen ST, Eitson JL, Caron R, et al. Genetic variation at IFNL4 influences extrahepatic interferon stimulated gene expression in chronic HCV patients. J Infect Dis 2017 Nov 20. doi: 10.1093/infdis/jix593. [Epub ahead of print]

33. Sansonno D, Russi S, Serviddio G, Conteduca V, D'Andrea G, Sansonno $\mathrm{L}$, et al. Interleukin 28B gene polymorphisms in hepatitis $\mathrm{C}$ virusrelated cryoglobulinemic vasculitis. J Rheumatol 2014;41:91-98.

34. Stättermayer AF, Rutter $K$, Beinhardt S, Scherzer TM, Stadlmayr A, Hofer $\mathrm{H}$, et al. Association of the IL28B genotype with insulin resistance in patients with chronic hepatitis C. J Hepatol 2012;57:492-498. 